\title{
HIGH VOLTAGE SHORT PULSES GENERATOR
}

\author{
M.G. Bakharev, B.I.Grishanov, F.V. Podgorny, \\ Budker Institute of Nuclear Physics, Novosibirsk, 630090, Russia
}

\begin{abstract}
High voltage short pulses generator is described. The generator is based on stepped pulse forming network (PFN). The generator forms pulses up to $50 \mathrm{kV}$ amplitude at a charging voltage of $25 \mathrm{kV}$. Pulse duration (full width half maximum FWHM) is $80 \mathrm{nsec}$, load resistance is $50 \mathrm{Ohm}$. Hydrogen high-voltage thyratron is used as a switch. The thyratron shorts $6.25 \mathrm{Ohm}$ end of the PFN. This generator is intend for fast injection and extraction systems of charged particle accelerators.
\end{abstract}

\section{INTRODUCTION}

In the spring of 1999 a decision was taken on the modernization of the VEPP-2M complex to increase the luminosity and the maximum achievable energy of up to $2 \mathrm{GeV}$. This will to improve the experiments on the collider essentially. The new project was named VEPP2000. The energy of injection is $900 \mathrm{MeV}$. The scheme of injection with a prekick of a stored beam is used. As the collider has got one ring, both the electron and the positron beams co-exist and simultaneously move in counter directions. The kickers operate in a traveling wave mode and affect the counter moving particles only. Every kicker has one asymmetrical $50-\mathrm{Ohm}$ strip lines and is fed by unipolar pulses [1].

The generators forms pulses in the traveling wave mode with an amplitude of up to $50 \mathrm{kV}, 80 \mathrm{~ns}$ pulse duration (FWHM) and $60 \mathrm{~ns}$ raise/fall time at a repetition rate of up to $1 \mathrm{~Hz}$. The circuit is based on a step artificial line as the PFN and a pulse hydrogen thyratron as the switch.

\section{PULSE TRANSFORMERS}

To form high voltage pulses various ways are used. The most usual method is, that a preliminary charged energy storage discharges through the switch to a matched load. If the energy storage is used a forming line, voltage amplitude on the load is equal to half of the line charge voltage. Sometimes it becomes diseconomical, when too high voltages are required. That is why the circuit with a double forming line is used rather often; this circuit frequently called as a Blumline-scheme. In this circuit a pulse amplitude is equal to the charge voltage. If the higher voltages are required, pulse transformers could be applied. Many works are attended to pulse transformers. A coaxial cable transformer is one of the types of the pulse transformer. A winding consists of several pieces of the coaxial cable, which is coiled on a ferromagnetic core. Braids of the cable pieces are connected in parallel and form a primary coil of the transformer. Inner conductors of the cable pieces are connected in series and form a secondary coil. A transformation ratio is equal to the cable pieces number.

Other type of the pulse transformer is the transformer on pieces of delay lines. The transformer represents the device consists of several delay lines, which are connected in parallel on inputs, and in series - on outputs. The transformer feeding pulse reaches the transformer output over delay time $t=1 / \mathrm{v}$ ( 1 - length of lines, $v-$ wave distribution speed). If the output loaded with $R=n \rho$, where $\rho$ - wave resistance of a single line, the voltage amplitude on the load becomes $n$ times more input pulse amplitude. There are also other types of pulse transformers [2]. As well as any transformers, they bring distortions.

\section{GENERATOR}

There is a circuit that allows to form theoretically rectangular pulse without tails, which amplitude exceeds the charge voltage. In a presented work the generator consists of a four-terminal forming circuit, a switch, a load R2 and a charging device. The energy, which has been stored in the all line pieces at a charge voltage $E$, completely dissipates on the load R2 during the pulse [3]. Figure 1 shows an equivalent circuit of such kind generator having four steps. The fourterminal forming circuit includes three long lines X1, X2, $\mathrm{X} 3$ and an additional line $\mathrm{X} 4$.

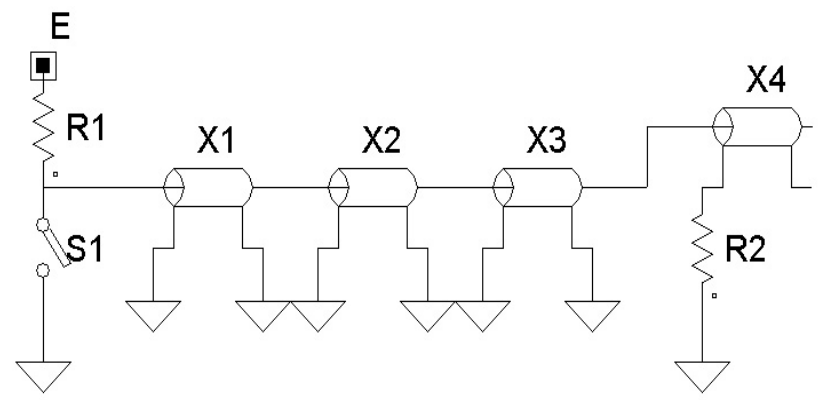

Figure 1: The equivalent circuit of the generator.

Wave resistances of each step $X_{k}$ are defined as follows:

$$
\begin{gathered}
X_{k}=\frac{k(k+1)}{n^{2}} R 2, \\
k=1,2, \ldots, n-1 \\
X_{n}=\frac{R 2}{n} \quad, \text { where } \\
\mathrm{k}-\text { is a line order number, } \\
\mathrm{n}-\text { is the step number. }
\end{gathered}
$$


We define the output voltage is equal to $n \times(0.5 \mathrm{E})$. The transformer ratio of this circuit is determined as a relation of output pulse amplitude to charge voltage. It is necessary to mention here, that both the single line and Blumline circuits represent special cases of such fourterminal circuit for one and two steps accordingly. It is possible to get the greater output voltage pulse by means of the step numbers increasing. A "clean" output pulse could be formed only using relations (1) and (2). Each step adds $0.5 \mathrm{E}$ at such conditions.

In the given work the generator of short high-voltage pulses, which is constructed by the technique described above, is presented.

The wave resistance of the lines are:

$$
\begin{aligned}
& \text { X1 - 6,25 Ohm, } \\
& \text { X2 - 18,75 Ohm, } \\
& \text { X3 - 37,5 Ohm, } \\
& \text { X4 - 12,5 Ohm. }
\end{aligned}
$$

All lines of the four-terminal forming circuit are executed as artificial lines. The necessary number of cells, which could be enough to get an acceptable value of oscillations at the top and tails, was chosen after simulation with program NL developed in BINP. Figure 3 shows the simulated pulse shape at 10 cells number in each line.

The design of the generator looks as follows: four consistently connected lines of the generator are placed in one common cylindrical pipe.

The inductance of the first line is realized as a metal tube, because of a small wave resistance of this line. In other lines inductance is carried out as spirals.

It is visible from Figure 4 that the common conductor is connected directly to the generator output, as the result the output voltage appears on it. Such design could be a source of the serious noises for the measuring and control equipments. There is a decision, when the additional line is installed into a coaxial structure. Such design is difficult for carrying out technically if the artificial lines are used.

To avoid the noise in the given design the capacity of a last cell of the line $\mathrm{X} 3$ is executed as a piece of a coaxial line with the wave resistance equals one of X3. An inner tube of this coax is used as a place for the additional line X4. As the result forming part of the generator has a completely shielded design. One end of it connects with the thyratron; another end connects with the load.

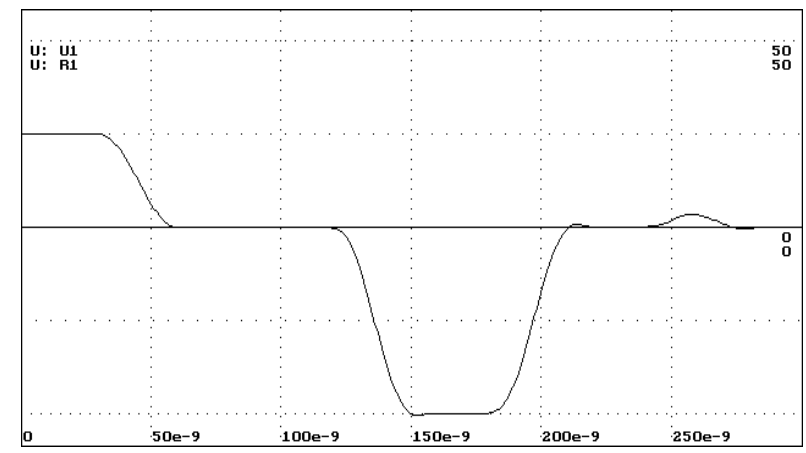

Figure 3: The simulated pulse.

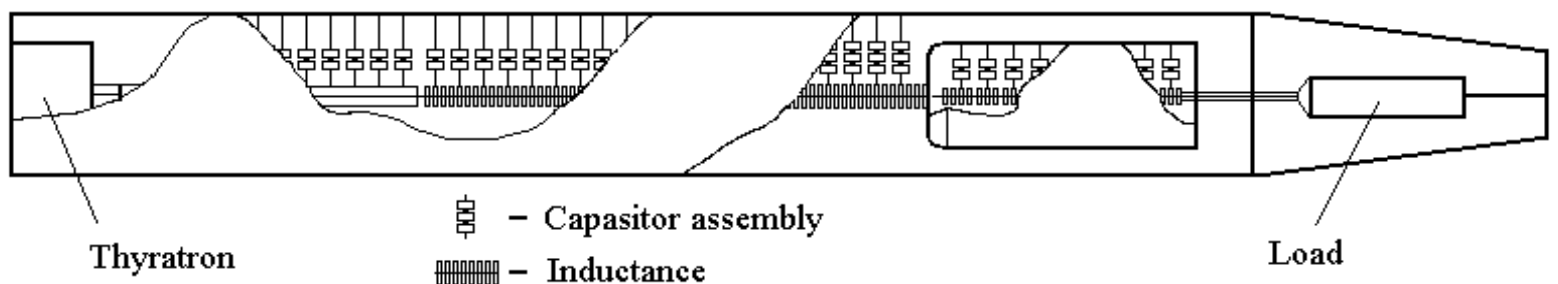

Figure 4: Cartoon of the generator prototype.
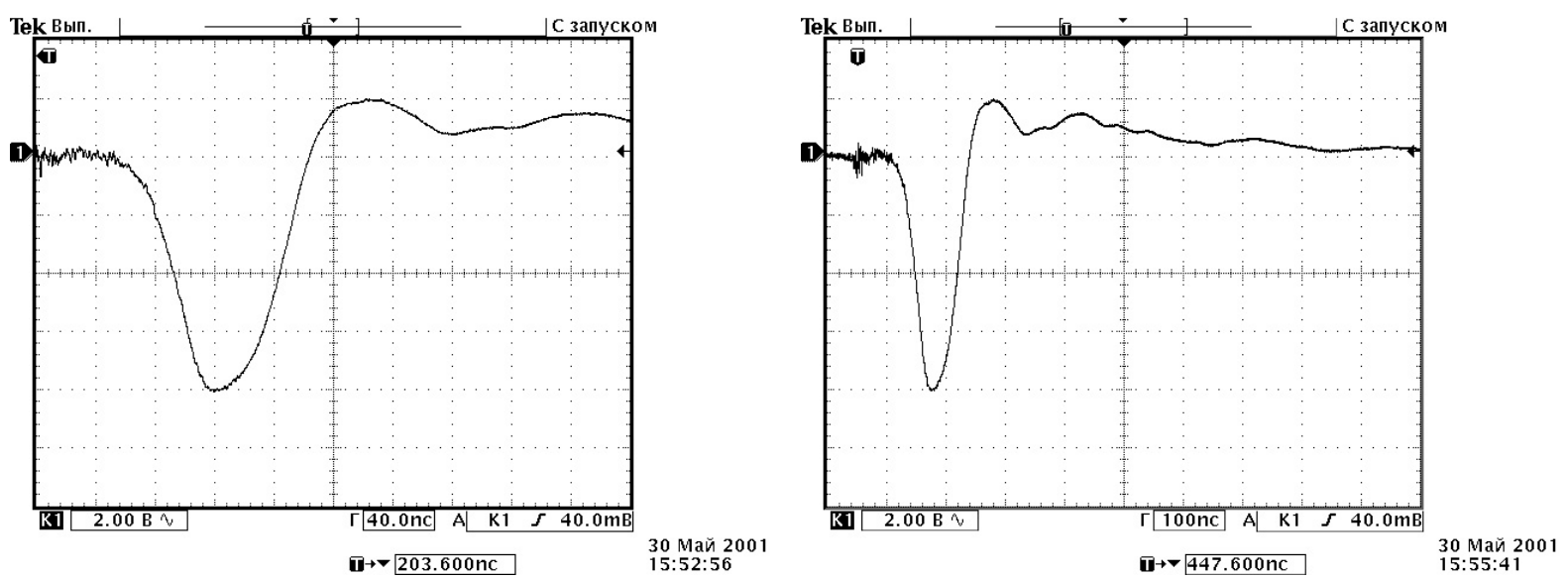

Figure 5: Output pulse from divider. 
To check a capacity to work of this circuit, a prototype was assemble with the following parameters:

Output pulse voltage

up to $50 \mathrm{kV}$

Pulse duration (FWHM)

$80 \mathrm{~ns}$

Repetition rate

$1 \mathrm{~Hz}$

The thyratron TGI1-1000/25 is used as the switch. The nominal rating of this unit allows to switch a current of $1000 \mathrm{~A}$ at $25 \mathrm{kV}$ of the anode voltage. We have an experience of using such type thyratrons at the current up to $4000 \mathrm{~A}$ and at $25 \mathrm{kV}$ at short pulses duration. Preliminary low voltage tests were done to check a possibility to form short pulses in principle. Then the prototype (Figure 4) was tested at the nominal voltage. The breakdown test was carried out, and breakdowns have appeared at $28 \mathrm{kV}$ of the charge voltage. The measurements of the output pulses were execute by a capacitor divider (it is not shown in Figure 4), using constructive capacities. Oscillograms from the capacitor divider are shown on Figure 5.

The transformer ratio can be increased yet more. The input impedance of such circuit (the same as the wave resistance of the line X1) loaded with R2 must be:

$$
X 1=\frac{2 R 2}{n^{2}}
$$

The circuit allows executing line $\mathrm{X} 1$ as several parallel lines with higher wave resistance. At that each line is switched separately by its own thyratron. Technical features of the thyratron synchronization at a parallel mode are described in the literature [4]. There are no problems concerned with the parallel work of the switches in the offered variant. Such decision proves to be more adequate in some cases.

\section{REFERENCES}

[1] Injection in the storage ring VEPP-2000. B. I. Grishanov, F. V. Podgorny. HEACC-2001.

[2] Mesjac G.A., Nasibov A.S., Kremnev V.V. Formirovanie nanosekundnih impulsov visokogo naprjazenija. Moskow, 1970.

[3] Spravochnik po impulsnoi tehnike. Kiev, Technika, 1971.

[4] Morygin L.A., Glebovich G.V. Nanosekyndnaja impulsnaja tehnika. Moskow, 1964. 